\title{
On the advancement of maintenance management towards Smart Maintenance in manufacturing
}

\author{
Macchi M., Roda I., Fumagalli L. \\ Politecnico di Milano, Piazza Leonardo da Vinci 32, Milano, Italy \\ \{marco.macchi; irene.roda; lucal.fumagalli\}@polimi.it
}

\begin{abstract}
The purpose of this work is to envision the future of maintenance without forgetting the past and present of maintenance practices. Indeed, there is a big potential for maintenance, fostered by the promises of the Fourth Industrial Revolution. At the same time, there is still a widespread evidence of the state of practices leading to assert that maintenance is not yet advanced as it would be expected. Thus, comparing the vision supported by advanced maintenance systems, through the concepts of E-maintenance, Internet of Things and Cyber Physical Systems, with the evidences on the state of practices collected based on a sample of more than 300 industrial plants, we come up with a reflection on the advancement of maintenance management towards Smart Maintenance.
\end{abstract}

Keywords: Smart Maintenance, Maturity assessment, Survey, Manufacturing, Industry 4.0

\section{$1 \quad$ Introduction}

In recent years, the maintenance management discipline is focusing on the role of maintenance to contribute to value for business [1]. The emerging perspective was that maintenance should not be viewed only at a narrow operational level representing an unavoidable cost; but it must be viewed in the long-term strategic context, integrating technical and commercial issues in an effective manner [2]. The strategic role of maintenance was remarked primarily by viewing it from the overall business perspective, pointing out that its effectiveness is a result of a multi-disciplinary approach [2][7]. Today, maintenance is seen as source of added-value, with key role for driving performance improvement [1]. This is an evolution of the past developments of maintenance. If we consider a short overview of history, attention to maintenance management has been growing along the years. At the beginning of $20^{\text {th }}$ century, before the First Industrial Revolution occurred, homemade production was just requiring Corrective Maintenance (CM). Only with mass production, the need for preventive maintenance programs was growing, mainly with the purpose to keep quality of processes and machines required by the production volume to be released to the market. This led to the growth of Time-Based Maintenance (TBM) practices. With the growing complexity of industrial plants and the awareness that TBM was not really a cost-effective solution 
when applied to complex physical assets, industrial development led to the introduction of diagnostics techniques: these were the first signs of a change, towards the introduction of the so-called Condition Based Maintenance (CBM) programs [8]. The approach used to attack problems in CBM was primarily built on technical insights, due to the measurement of the physical parameters used as symptoms of asset degradation; this brought a wide set of technical specializations, such as in vibration analysis, oil analysis, thermography, etc.... In the meanwhile, two relevant maintenance success stories occurred, originally in specific industry sectors, leading to the two well-known methodologies of RCM (Reliability Centred Maintenance) [9] and TPM (Total Productive Maintenance) [10]. The former had its origin in sectors such as aerospace and aeronautics, where reliability and risk analysis were relevant for safety reasons. The latter is historically associated to the Toyota Production System, related to the needs arising in Just in Time, Total Quality Management and continuous improvement practices. It is worth observing that such developments had a common feature: they were bringing knowledge on engineering methodologies (i.e. FMECA, FTA, Pareto analysis, 5 whys, etc.), providing means to identify and prioritize maintenance problems, and to subsequently discover improvement solutions. Especially after TPM, a wide literature emerged regarding the so-called lean production, focused on organizational aspects. Since that time, maintenance is seen as a relevant function called, thanks to its technical authority, to participate to problem solving, contributing to operational excellence.

All the developments mentioned above had in common, as underlying concept, a blend of technical, engineering and organizational factors. Information and Communication Technologies (ICTs) came out only partially during this historical development. Around the 90ies, this happened with the development of Computerised Maintenance Management Systems (CMMS), used to cover the needs for controlling maintenance management and managing the information related to the maintenance activities. Around the 90ies, the first ideas of exploiting Internet for providing tele-maintenance services emerged, typically pushed by Original Equipment Manufacturers (OEMs). Nowadays, we are observing a new technology wave, where OEMs play, of course, a role. Indeed, it is remarkable how the vision of Industry 4.0 pushes the role of ICTs as primary technology enabler for enhanced maintenance practices. Given the vision of Industry 4.0, the present paper aims at reflecting on the expected trends, starting from state of practice in industry. It may be appropriate to address a balanced development due, on one hand, to the "technology-push" for the future and, on the other hand, to the "process-pull" resulting from past and present status.

To this end, section 2 provides a vision of the "technology-push", with the actual and future developments, primarily in relationship to Cyber Physical Systems (CPS) applied to maintenance. Section 3 looks at the missing or poor practices observed in the maintenance processes in manufacturing industry. This evidence is based on a benchmarking activity carried out, across Italy, in the context of the Observatory on Technologies and Services for Maintenance (TeSeM) of the School of Management of Politecnico di Milano. Section 4 reflects on an overall approach to master the deployment of the technology promise in an existent company. Conclusions envision the remarks for future research works on this matter, and the subsequently planned activities in the context of the current TeSeM's yearly research. 


\section{Vision on the different technologies}

Industry 4.0 is leading to think of maintenance as a relevant function for strategic improvement of Industry 4.0-like solutions. The Fourth Industrial Revolution enhances what past visions, as E-maintenance and Intelligent Maintenance Systems (IMS), have achieved, employing the related key enabling technologies (Internet of Things, Cyber Physical Systems, etc.) for building advanced maintenance systems [11]-[13]. Above all, a blend of ICT- and plant automation-enabled solutions is expected to grow, thus empowering the traditional features of maintenance management, based on the blend of technical, engineering and organizational factors. Considering an overall strategic perspective of Industry 4.0, that would better consider a "process pull", the short/middle term vision considers a technology push. The technology push moves around some main drivers, related with different technologies that can be considered for maintenance activities and in particular:

- Technologies for maintenance analysis that can improve the diagnostic and prognostic capabilities of the actual CBM system.

- Technologies for communication and cloud computing that can guarantee: on one side the deployment of sensor network that can push the creation of communication backbone within the plants or among plants for the deployment of maintenance services (also considering OEM perspective); on the other side, the diffusion of maintenance analysis solutions, guaranteeing remote activity for maintenance engineering.

- Technologies for operator enhancement, such as new information systems, new portable devices and other new means to consider the operator in the loop. To this category belongs also the solution for augmented and virtual reality that are emerging, even if not yet applied extensively to the maintenance processes.

- Technologies for data analytics are pushing a new way to analyse maintenance data, paving the way also for new engineering profile, namely closer to mathematical engineers than to mechanical or electrical engineers for maintenance analysis.

\section{State of practice}

The analysis of the current state of practice is presented referring to the data collected by the research of the TeSeM Observatory, that issued the first research in 2013, aiming to implement a maturity level analysis on the current maintenance management practices of production companies in Italy. Three hundred industrial companies in different industrial sectors (mechanical, food, chemical-pharmaceutical and others) and of different sizes (34\% SMEs and 66\% Big) compose the reference sample. The research is based on a maintenance maturity assessment method developed by the TeSeM and implemented through a questionnaire [14]. The method allows assessing the maintenance maturity both as a synthetic index - in the remainder general maturity index (GMI), for measuring the general Maturity Level (ML) - and a set of component indexes accordingly with the need to measure the managerial, organizational and technological capabilities of the maintenance department. A scale of five maturity levels is used. ML1 is the initial level (meaning that the process is weakly controlled, or not controlled at all). 
ML5 is the optimizing one (meaning that process is managed by ensuring the continuous improvement; causes of defects and problems in the processes are identified and actions taken in order to prevent problems from occurring in the future) [14]. A general overview regarding the GMI in the Italian industry nowadays is given in Fig.1. Almost $50 \%$ of the analyzed companies are still presenting a GMI at ML1 and ML2, giving a first general indication about a great area for improvement in the current practices.

Fig. 1. Distribution of General Maturity Index of the sample

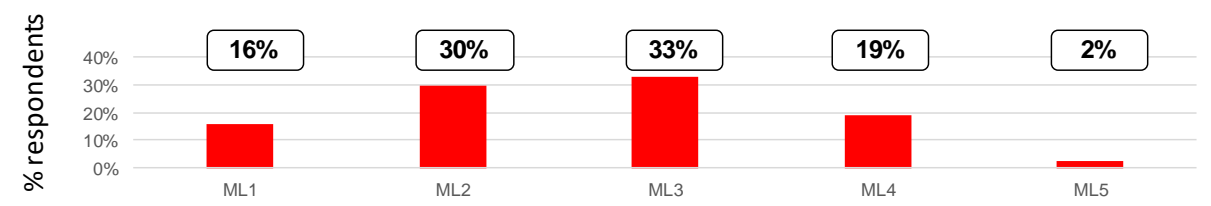

When considering in detail the current state of practice on CBM implementations, the statistics are the following. Only few companies (around 30\% in each considered industrial sector) have monitoring / inspection tools connected with maintenance decision support tools (i.e. diagnostics and prognostics).

Fig. 2. Statistics on the connection of monitoring/inspecting tools with fault diagnosis and/or prognostic tools within the plants in the sample, divided by sector
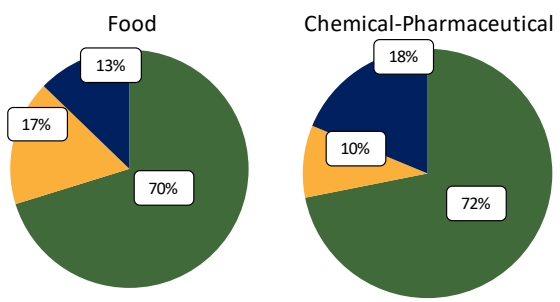

LEGENDA:

No

Yes (remotely)

- Yes (on board the plant/the tool)

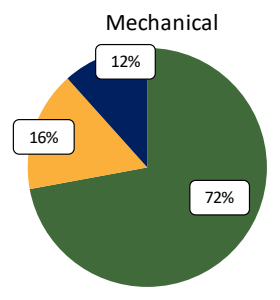

Moreover, only few companies implemented a "network integration" of the tools used for CBM in order to provide a remote connection/access to the maintenance information system. Only the Chemical-Pharmaceutical sector demonstrates a reasonable (even if not so high) tendency to network integration (28\%) (Fig.3).

Finally, looking at how companies analyse data obtained from inspections/monitoring and how they make decisions about CBM, in the majority of cases, data analytics is not adequately used in order to support management decisions. In figure, about $70 \%$ of companies in each sector do not implement in-depth analysis using specialist software; present no systematic filing of data and decisions are based mainly on information available at the last inspection/most recent monitoring. Only a limited number of cases is featuring advanced uses of data analytics for management decisions. Those are the cases where specialist software are used and data arecorded in the CMMS and/or other specialist tools for maintenance engineering. 
Fig. 3. Statistics on "network integration" to provide a remote connection/access to the maintenance information system within the plants in the sample, divided by sector
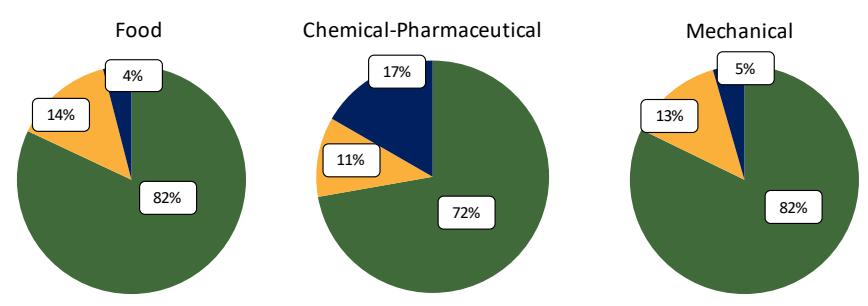

$$
\begin{aligned}
& \text { LEGENDA: } \\
& \text { No } \\
& \text { Yes (in located network of one factory) } \\
& \text { - Yes (in network of several factors) }
\end{aligned}
$$

Fig. 4. Statistics on how companies analyse data obtained from inspections/monitoring and how they make decisions about condition-based maintenance
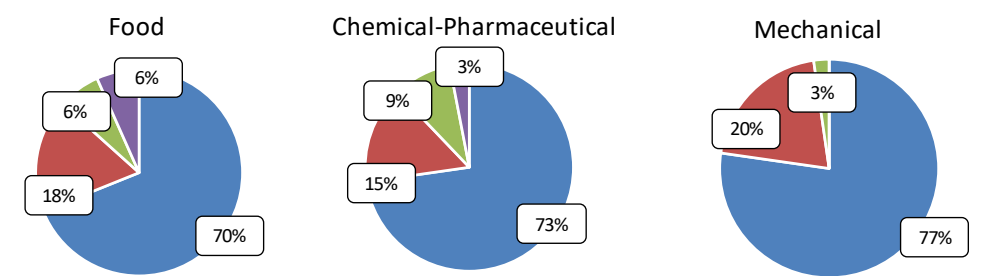

No in-depth analysis using specialist software; no systematic filing of data; decision based mainly on information available at the last inspection/most recent monitoring

Thorough analyses of the conditions using specialist software to support decisions on a mainly technical basis

Thorough analyses of the conditions using specialist software to support decisions on a mainly technical basis
Thorough analyses of the conditions using specialist software, recording data in the CMMS and/or other specialist tools for maintenance engineering, in order better to define the frequencies of the maintenance plan

Thorough analyses of the conditions using specialist software, recording data in the CMMS and/or other specialist tools for maintenance engineering, in order (i) better to define the frequencies of the maintenance plan and (ii) to support decisions on coordination between the production and maintenance departments as regards planning plant shutdowns based on the predicted time remaining before a fault

\section{On the advancement towards Smart Maintenance}

Given the current state of practice, we may deduce that problems still exist that could prevent or slow down the capability to digitize maintenance. It is evident that a profound distance exists between the vision suggested by Industry 4.0 and the CBM status in different industries. Today, the data-to-information and -to-knowledge-conversion are still fragmented and incomplete, emerging from the statistics above shown (see fig. $2,3,4)$. Wastes are subsequently generated during maintenance operations, while reduced improvements (compared to the desired targets) in terms of, e.g., Overall Equipment Effectiveness can be expected. Overall, a reduced cost-effectiveness of the CBM programs is the outcome. Besides, it is worth remarking that cost-effectiveness depends on the type of resource required by CBM activities. As professionals with high-level qualification, experience and use of sophisticated equipment and decision models are often required in case of critical assets not manageable using resources and competences inside a company, an increase of cost is directly associated to the CBM program 
execution. Relating to the technology promise, the question that arises is: "can CPS applied to maintenance enable to reduce the drawbacks of the actual CBM status?" CPSs are promising at least two major properties: a seamless integration and an enhanced intelligence in maintenance activities, that are the two main levers to improve the actual status. CPS-based integration is a mechanism to limit the fragmentation, connecting the "islands" of maintenance specialists through CPS-ization (and, eventually, subsequent "social networks" of machines, featuring capabilities for analysing different degradation processes and failure modes). Depending on the CPS-ization, different levels of integrations can be defined into the management system, enabling to achieve the degree of completeness that is required for a CBM process by the asset criticalities. Indeed, not always a full integration is required for what concern the CBM program execution, i.e. integration from state detection to health and prognostics assessment and even maintenance within production scheduling. Seamless integration would help defining the integration that best fits the requirements due to the asset criticalities. Intelligence will eventually enable better accuracy in the functions executed along the CBM process. In fact, as each function of the CBM process is executed based on proper models bringing intelligence - either statistical, physical, soft computing or operations research models -, it would result in higher expectation for achieving the Overall Equipment Effectiveness's target. In this regard, CPS-ization may be also a relevant means to bring on-line all engineering knowledge - as intelligence - even considering methodologies - as FMECA, HAZOP, etc. - traditionally adopted in an off-line fashion for designing industrial plants, processes and management policies.

If the technology promise is convincing for CBM operations, the question that now arises is: "can an existent Maintenance Organization rely on new technologies as CPSs to reduce the drawbacks of the actual CBM status?" Maintenance organization is a relevant issue for digitization: it is an evidence achieved during the first workshop of a series of the new research, in the context of TeSeM Observatory, on digital readiness of maintenance. In this regard, four organizational criteria came out during the workshop: i) maintenance engineering not only comprises engineering knowledge, but it is an organizational unit that can promote and support the implementation of innovation projects in asset management; ii) utilizing new technologies should be supported by the developments of people that are using them in their tasks, as their tasks are changing; iii) change management requires a participative approach from different levels in the organization, only so real commitment to the change is possible; iv) global organization could be a relevant way to strengthen the probability of success of a digitization process, as it may enable the identification of different areas of implementation in various plants worldwide, and global sharing of best practices growing in such areas.

Overall, CPS in maintenance requires more than technology to enable the construction of an advanced maintenance management system. Digitization requires to master technology and organization, in order to move forward to build a Smart Maintenance system. Therefore, an organizational perspective should be taken, covering different aspects, as e.g. maintenance activities and tasks in the organization, decisions and roles to be supported, skills etc. A particular need that seems to be relevant in this end is the focus on the processes executed by maintenance. Fig.5 represents maintenance processes, derived - by translation and minor adaptations - from the UNI norm [15]. 


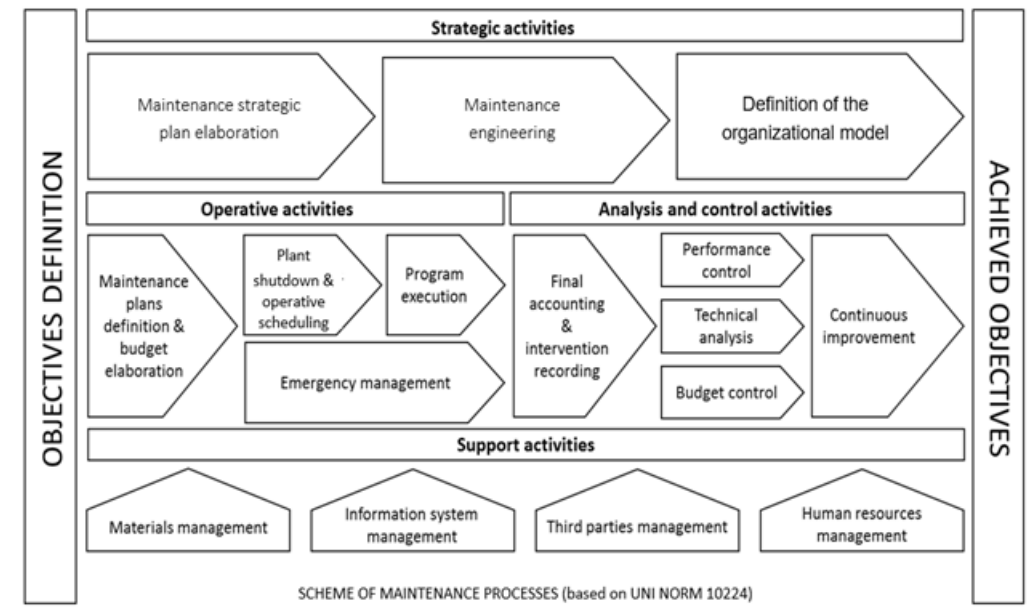

Fig. 5. Maintenance processes [UNI 10224:2007]

A process map can support targeting areas for digitization in maintenance, as a lever to contribute to business value. In this sense, it allows not only to consider the "technology push" - due the potentials of different technologies envisioned by Industry 4.0 - but to drive decisions based on a strategic prioritization of maintenance processes, according to the fact that processes and their gaps pulls the need for technology improvement, i.e. "process-pull". In this reflection, we assume that a company may look at such processes both considering their status (and thus gaps with respect to the Industry 4.0 vision) and their prominence for the business value. Maturity assessment of maintenance processes as well as the subsequent understanding of their digital readiness appear relevant research topics to connect the technology promise with the maintenance actual needs.

\section{Conclusions}

This paper initiated a reflection that is at the background of the activities carried on during the past and present yearly researches by the TeSeM Observatory of the School of Management of Politecnico di Milano. Based on the actual results reported in the paper, we can remark that benchmarking of maintenance management practices appears to be a relevant tool to assess the actual maintenance status. It enables to support the identification of gaps with respect to the Industry 4.0 vision. This can be relevant to assess the real capability to plan for digitization initiatives, verifying the digital readiness of maintenance. Overall, this approach enables to think of a balanced development due, on one hand, to the "technology-push" for the future and, on the other hand, to the "process-pull" resulting from past and present status. In fact, it is difficult for a company to focus a decision when a number of requests comes from technology providers, that apparently sell the best of Industry 4.0-solutions. A strategic assessment of needs 
and gaps in a company enable a clearer view of the need of maintenance in the contingency of its industry. The yearly research of TeSeM will develop a number of empirical studies, with the purpose to develop a roadmap of digitization for maintenance, providing a balanced perspective between "technology-push" and "process pull".

\section{Acknowledgments}

The research was performed within the scope of TeSeM Observatory (tesem.net)

\section{$6 \quad$ References}

[1] M. Holgado, M. Macchi, L. Fumagalli, "Maintenance business model: a concept for driving performance improvement," Int. J. Strateg. Eng. Asset Manag., 2015.

[2] D. N. P. Murthy, A. Atrens, and J. A. Eccleston, "Strategic maintenance management," J. Qual. Maint. Eng., vol. 8, no. 4, pp. 287 - 305, 2002.

[3] U. Al-Turki, “A framework for strategic planning in maintenance," J. Qual. Maint. Eng., vol. 17 , no. 2, pp. 150-162, 2011.

[4] K. El-Akruti, "The strategic role of engineering asset management in capital intensive organisations," University of Wollongong, 2012.

[5] J. P. Liyanage, "State of the art and emerging trends in operations and maintenance of offshore oil and gas production facilities: Some experiences and observations," Int. J. Autom. Comput., vol. 7, no. 2, pp. 137-145, 2010.

[6] A. H. C. Tsang, "Strategic dimensions of maintenance management," J. Qual. Maint. Eng., vol. 8, no. 1, pp. 7-39, 2002.

[7] G. Waeyenbergh, L. Pintelon, "A framework for maintenance concept development," Int. J. Prod. Econ., vol. 77, no. April 2000, pp. 299-313, 2002.

[8] A. K. S. Jardine and A. H. C. Tsang, Maintenance, replacement and reliability: theory and applications, Boca Raton. 2006.

[9] M. Rausand, "Reliability centered maintenance," Reliab. Eng. Syst. Saf., 1998.

[10] I. P. S. Ahuja and J. S. Khamba, "Total productive maintenance: literature review and directions," Int. J. Qual. Reliab. Manag., vol. 25, no. 7, 2008.

[11] A. J. Guillén, A. Crespo, M. Macchi, J. Gómez, "On the role of Prognostics and Health Management in advanced maintenance systems," Prod. Plan. Control, 2016.

[12] M. Emmanoulidis C., Iung B., Macchi and F. Peres, "Foreword Proceedings of the 3RD IFAC WORKSHOP ON ADVANCED MAINTENANCE ENGINEERING, SERVICES AND TECHNOLOGY," in AMEST 2016,.

[13] J. Lee, B. Bagheri, and H.-A. Kao, "A Cyber - Physical Systems architecture for Industry 4 . 0 - based manufacturing systems," vol. 3, pp. 18-23, 2014.

[14] M. Macchi and L. Fumagalli, "A maintenance maturity assessment method for the manufacturing industry,” J. Qual. Maint. Eng., vol. 19, pp. 295-315, 2013.

[15] "UNI 10224:2007 Maintenance - Process, Sub-processes And Main Activities Fundamental Principles.” UNI Italian Standards. 